Ergod. Th. \& Dynam. Sys. (1983), 3, 167-185

Printed in Great Britain

\title{
Which groups act distally?
}

\author{
HERBERT ABELS \\ Fakultät für Mathematik, Universität Bielefeld, D-48 Bielefeld 1, West Germany
}

(Received 23 March 1982 and revised 18 September 1982)

Abstract. The following question is discussed: which locally compact topological groups have an effective distal action on some compact metrizable space?

\section{Introduction}

Among the best understood actions of locally compact groups on compact spaces are the distal ones. By a fundamental theorem of Furstenberg [7] they can be built up from a one point space by a possibly transfinite sequence of so-called isometric extensions. In this paper we deal with the question of which groups have an effective distal action on some compact space. We give a necessary condition in terms of the existence of certain finite dimensional unitary representations on normal subgroups.

As an application of our main theorem we show in $\$ 4$ that the discrete 'rational $a x+b$-group' does not admit an effective distal action, although most subgroups do (examples 4.3 and 4.6).

We also prove, in $\S 3$, that a connected locally compact topological group admits an effective distal action iff it has polynomial growth (theorem 3.5). The necessity follows from the above condition, and has also been proved in [12]. In order to prove the sufficiency we show that every connected Lie group of polynomial growth can be embedded as a closed normal subgroup into a compact extension of a connected nilpotent Lie group. This result may be of independent interest. It is an outcome of the author's study of distal automorphism groups of Lie groups [2].

Basic to our approach is the result proved in $\S 1$ that for every isometric extension $X \rightarrow Y$ there is an associated principal fibre bundle $E \rightarrow Y$, which is also an isometric extension. We obtain our necessary condition for distality by looking at the homomorphism from the ineffective kernel of $Y$ to the structure group of the principal fibre bundle $E \rightarrow Y$ (see $\S 2$ ).

There is an analogous theory in the measure theoretic case for ergodic actions with generalized discrete spectrum, where the analogue of our principal fibre bundle can simply be described by a cocycle [14]. The result of Moore and Zimmer [12] says that an almost connected group $G$ admits an ergodic action with generalized discrete spectrum iff $G$ has polynomial growth. It is the measure theoretic analogue of our theorem 3.5, one part of which is already in [12].

\section{Distal actions and isometric extensions}

In this section we recall the basic definitions and results on distal actions. We also study isometric extensions in some detail and prove the existence of an associated principal fibre bundle with compact structure group and fibre. 
Let $G$ be a locally compact topological group. By a (compact) $G$-space we mean a (compact) metric space $(X, d)$ together with a continuous action of $G$ on $X$, i.e. a continuous mapping

$$
G \times X \rightarrow X, \quad(g, x) \rightarrow g x
$$

with the usual properties. A compact $G$-space $X$ is called distal if for any two points $x$ and $y, x \neq y$, of $X$ there is an $\varepsilon>0$ such that

$$
d(g x, g y)>\varepsilon \quad \text { for every } g \in G .
$$

A $G$-space $X$ is called minimal if there are no closed $G$-invariant subspaces except $\varnothing$ and $X$; equivalently, if every $G$-orbit is dense. A theorem of Ellis [6], [7] says that in a compact distal $G$-space all orbit closures are minimal (distal) $G$-spaces, thus reducing the study of distal $G$-spaces to minimal ones.

In order to state the fundamental structure theorem on compact distal $G$-spaces we recall Furstenberg's definition of an isometric extension.

Definition 1.1. Let $X$ and $Y$ be compact $G$-spaces and let $\pi: X \rightarrow Y$ be a $G$-map. The map $\pi$ is called an isometric extension of $Y$ if there is

(a) a non-empty compact metric space $\left(M, \rho_{M}\right)$, which is homogeneous, i.e. the group of its isometries acts transitively on $M$, and

(b) a fibrewise metric $\rho$ for $\pi$; i.e. a continuous map $\rho$ defined on the fibred product

$$
X \times_{Y} X=\left\{\left(x_{1}, x_{2}\right): \pi\left(x_{1}\right)=\pi\left(x_{2}\right)\right\}
$$

which is, when restricted to any fibre $X_{y}=\pi^{-1}(y)$ a metric on $X_{y}$, such that

(c) $\left(X_{y},\left.\rho\right|_{X_{y} \times X_{y}}\right)$ is isometric to $\left(M, \rho_{M}\right)$ for every $y \in Y$, and

(d) $G$ leaves the fibrewise metric $\rho$ invariant, i.e. for $x_{1}$ and $x_{2}$ in $X$ with $\pi\left(x_{1}\right)=$ $\pi\left(x_{2}\right)$ we have

$$
\rho\left(g x_{1}, g x_{2}\right)=\rho\left(x_{1}, x_{2}\right) .
$$

There is the following fundamental result of Furstenberg [7].

THEOREM 1.2. A non-empty compact minimal $G$-space $X$ is distal iff there is an ordinal $\alpha$ and an inverse system of compact $G$-spaces $X_{\beta}, \beta \leq \alpha$, and surjective $G$-maps $X_{\beta} \rightarrow X_{\beta^{\prime}}$, for $\beta \geq \beta^{\prime}$, indexed by the ordinals $\beta \leq \alpha$ such that the following conditions hold:

(a) $X_{\alpha}=X$ and $X_{0}$ is the one point space, for 0 the least ordinal;

(b) $X_{\beta+1} \rightarrow X_{\beta}$ is an isometric extension for $\beta<\alpha$;

(c) if $\beta \leq \alpha$ is a limit ordinal, $X_{\beta}=\varliminf_{\gamma<\beta} X_{\gamma}$.

A system as above is called a quasi-isometric system of order $\alpha$ for $X$. Given a distal $G$-space $X$, the order of $X$ is the least ordinal $\alpha$ such that there is a quasi-isometric system of order $\alpha$ for $X$. In this case we also call $X$ an $\alpha$-distal $G$-space.

Furstenberg's theorem reduces the study of compact minimal distal $G$-spaces to isometric extensions. We shall study them in some detail. Our main tool is the associated principal fibre bundle.

Let us recall the notion of the Ellis semigroup. Let $X$ be a compact space. The set $X^{X}$ of all-not necessarily continuous-maps from $X$ to $X$ is a compact topological space with respect to the topology of pointwise convergence and a 
semigroup with respect to composition of mappings. If $X$ is a compact $G$-space, the action gives a continuous homomorphism $G \rightarrow X^{X}$. The Ellis semigroup, $E(X)$, is, by definition, the closure in $X^{X}$ of the image of this homomorphism, i.e. a map $t: X \rightarrow X$ is in $E(X)$ iff there is a net $g_{i}$ of elements in $G$ such that

$$
t(x)=\lim g_{i}(x) \quad \text { for every } x \in X .
$$

Obviously $E(X)$ is a compact topological space and one can show it is a subsemigroup of $X^{\boldsymbol{X}}$. But neither are the elements of $E(X)$ continuous maps, nor is the composition map

$$
E(X) \times E(X) \rightarrow E(X)
$$

continuous, in general. One has only the following obvious continuity properties. For fixed $t \in E(X)$, the right translation map

$$
E(X) \rightarrow E(X), \quad s \mapsto s t,
$$

is continuous. For a fixed map $t \in E(X)$ the left translation map

$$
E(X) \rightarrow E(X), \quad s \mapsto t s,
$$

is continuous, if $t$ is continuous.

Basic to our approach is the following continuity property of isometric extensions (cf. [10]).

LemMa 1.3. Let $\pi: X \rightarrow Y$ be an isometric extension of compact $G$-spaces. Let $y$ be a point of $Y$ and define

$$
\bar{I}_{y}=\left\{t \in E(X): t\left(X_{y}\right) \subset X_{y}\right\} .
$$

Then for every net $t_{i}$ converging to $t$ in $E(X)$ and every net $s_{i}$ in $\bar{I}_{y}$ converging to $s \in \bar{I}_{y}$ the net $t_{i} s_{i} x$ converges to tsx for every $x \in X_{y}$.

Proof. Let us clarify a technical point first. We have two metrics defined for pairs of points $x_{1}, x_{2}$ of $X$ sitting over the same point of $Y$, the metric $d$ of $X$ and the fibrewise metric $\rho$. We claim that the corresponding concepts of being at a small distance coincide, i.e. given $\varepsilon>0$ there is a $\delta>0$ such that for every pair of points $x_{1}, x_{2}$ in $X$ with $\pi\left(x_{1}\right)=\pi\left(x_{2}\right)$ and $d\left(x_{1}, x_{2}\right)<\delta$ we have $\rho\left(x_{1}, x_{2}\right)<\varepsilon$ and vice versa. The proof is a straightforward contradiction argument using compactness.

We now prove the lemma. Since every $g \in G$ maps fibres of $\pi$ into fibres of $\pi$ and leaves the fibrewise metric $\rho$ invariant, so does every element $t \in E(X)$. Hence, with the notation of the lemma,

Hence

$$
\rho\left(t_{i} s_{i} x, t_{i} s x\right)=\rho\left(s_{i} x, s x\right) \rightarrow 0 .
$$

$$
d\left(t_{i} s_{i} x, t s x\right) \leq d\left(t_{i} s_{i} x, t_{i} s x\right)+d\left(t_{i} s x, t s x\right) \rightarrow 0,
$$

because the first summand tends to zero by the remark above and the second one does since the $t_{i}$ converge to $t$ pointwise.

Let $\pi: X \rightarrow Y$ be a surjective $G$-map of compact $G$-spaces. Then every element $t \in E(X)$ maps fibres of $\pi$ to fibres of $\pi$, hence induces a map $\pi_{*}(t): Y \rightarrow Y$ defined by

$$
\pi_{*}(t) \pi(x)=\pi(t x) .
$$


So $\pi$ induces a homomorphism $\pi_{*}: E(X) \rightarrow E(Y)$, which is continuous, obviously, and surjective, since $X$ is compact.

We now define the principal fibre bundle associated to an isometric extension. Let $\pi: X \rightarrow Y$ be a map of compact $G$-spaces. Define $I(y, z)$ to be the set of maps $f$ of the fibre $\boldsymbol{X}_{\boldsymbol{y}}$ to the fibre $\boldsymbol{X}_{z}$ induced by elements of $E(\boldsymbol{X})$, i.e. a map $f: \boldsymbol{X}_{\mathbf{y}} \rightarrow \boldsymbol{X}_{z}$ belongs to $I(y, z)$ iff there is an element $t \in E(X)$ such that $\pi_{*}(t) y=z$ and the induced map $t_{y}: X_{y} \rightarrow X_{z}$ 'is $f$. Let us fix a point $y_{0}$ of $Y$. Endow

$$
I\left(y_{0}, Y\right)=\bigcup_{y \in Y} I\left(y_{0}, y\right)
$$

considered as a space of mappings from $X_{y_{0}}$ to $X$, with the topology of uniform convergence. This is our bundle.

Proposition 1.4. Let $\pi: X \rightarrow Y$ be an isometric extension of a compact minimal G-space $Y$.

(a) $I=I\left(y_{0}, Y\right)$ is a compact metric space. The map $\beta: I \rightarrow Y$, given by

$$
\boldsymbol{\beta}\left(I\left(y_{0}, y\right)\right)=y,
$$

is continuous. On $I$, regarded as a space of mappings from $X_{y_{0}}$ to $X$, the topology of pointwise convergence and of uniform convergence coincide.

(b) (Principal fibre bundle.) $I\left(y_{0}, y_{0}\right)=: K$ is a compact topological group. There is a right action of $K$ on $I$, given by composition. This action is continuous, free and its orbits are the fibres of $\beta$.

(c) (Action of $E(X)$.) There is a left action of $E(X)$ on I given by composition. For every $y \in Y$ the restricted action map

$$
E(X) \times I\left(y_{0}, y\right) \rightarrow I\left(y_{0}, Y\right)
$$

is continuous. The orbit of any element of $I$ is $I$.

(d) (Action of $G$.) There is a left action of $G$ on $I$, given by composition. This action is continuous. The map $\beta$ is an isometric extension of $Y$ with fibre $K$. The $G$-space $I$ is a minimal $G$-space. Its Ellis semigroup is the image of $E(X)$ under the homomorphism $E(X) \rightarrow I^{I}$ given by the action in (c).

(e) (Induced bundle $=X$.) The map

$$
I \times_{K} X_{y_{0}}=I\left(y_{0}, Y\right) \times_{I\left(y_{0}, y_{0}\right)} X_{y_{0}} \rightarrow X, \quad[t, x] \mapsto t x,
$$

is a G-homeomorphism.

(f) (Independence of $y_{0}$.) Let $y_{1}$ be a point of $Y$ and let $t$ be an element of $I\left(y_{1}, y_{0}\right)$. Then right composition with $t$ defines an $E(X)$-equivariant homeomorphism $I\left(y_{0}, Y\right) \rightarrow I\left(y_{1}, Y\right)$.

Proof. Let us write $I$ for $I\left(y_{0}, Y\right)$.

(a) A metric on $I$ is defined by

$$
d_{I}\left(t_{1}, t_{2}\right)=\sup _{x \in X_{y_{0}}} d\left(t_{1}(x), t_{2}(x)\right) .
$$

By the Ascoli-Arzela theorem, $I$ regarded as a space of maps from $X_{y_{0}}$ to $X$, is compact if it is equicontinuous. But for $t \in I$ and $x_{1}, x_{2}$ in $X_{y_{0}}$ we have

$$
\rho\left(t x_{1}, t x_{2}\right)=\rho\left(x_{1}, x_{2}\right),
$$


which implies equicontinuity by the remark at the beginning of the proof of lemma 1.3 concerning the comparison of $\rho$ and $d$. Finally, let $I_{u}$ and $I_{p}$ be the set $I$ with the topology of uniform and pointwise convergence, respectively. The identity map $I_{u} \rightarrow I_{p}$ is a continuous bijective map from a compact space onto a Hausdorff space, hence a homeomorphism. So the two topologies coincide on $I$.

(b) The group of all isometries of $\left(X_{y_{0}}, \rho\right)$, endowed with the topology of uniform or equivalently, pointwise-convergence, is a compact topological group. This is well known and can also be proved like 1.3, 1.4(a) and 1.9. So $I\left(y_{0}, y_{0}\right)$ is a closed subsemigroup of a compact topological group, hence a topological group. The continuity of the action of $I\left(y_{0}, y_{0}\right)$ on $I$ follows from lemma 1.3, in view of the fact that uniform convergence and pointwise convergence coincide.

Since $Y$ is a minimal $G$-space, there are, for every $y \in Y$, elements $f_{1}$ and $g_{1}$ in $E(Y)$ such that $f_{1}\left(y_{0}\right)=y$ and $g_{1}(y)=y_{0}$. Since $\pi_{*}: E(X) \rightarrow E(Y)$ is surjective, there are elements $t \in I\left(y_{0}, y\right)$ and $s \in I\left(y, y_{0}\right)$. Their composite $s \circ t \in I\left(y_{0}, y_{0}\right)$ has an inverse. So we may assume that $s$ and $t$ are inverses of each other. So we have proved two things: every fibre of $\beta$ is non-empty; every element of $I\left(y_{0}, y\right)$ has an inverse in $I\left(y, y_{0}\right)$. It is now clear that the action of $I\left(y_{0}, y_{0}\right)$ on $I$ is free and that its orbits are the fibres of $\beta$.

Note that the proof of these two claims is easier in the case where $Y$ is a distal $G$-space, since then $E(X)$ and $E(Y)$ are groups, by a theorem of Ellis.

(c) To prove continuity of $E(X) \times I\left(y_{0}, y\right) \rightarrow I$, take an element $u \in I\left(y_{0}, y\right)$. The map

$$
L_{u}: I\left(y_{0}, y_{0}\right) \rightarrow I\left(y_{0}, y\right), \quad s \mapsto u \circ s,
$$

is a bijective continuous map of compact spaces, by (b), hence a homeomorphism. Now let $u s_{i}$ be a net in $I\left(y_{0}, y\right)$ converging to $u s$, and let $t_{i}$ be a net in $E(X)$ converging to $t$, then the net $s_{i}$ in $I\left(y_{0}, y_{0}\right)$ converges to $s$. Let $v$ be an element of $E(X)$ that restricts to $u \in I\left(y_{0}, y\right)$. Then $t_{i} v \rightarrow t v$, by continuity of right translations in $E(X)$, and finally

$$
t_{i}\left(u s_{i}\right)=\left(t_{i} v\right) s_{i} \rightarrow(t v) s=t(u s)
$$

by lemma 1.3 , which proves continuity of $E(X) \times I\left(y_{0}, y\right) \rightarrow I$.

The $E(X)$-orbit of any element $t \in I\left(y_{0}, y_{1}\right)$ contains the identity element $e$ of $I\left(y_{0}, y_{0}\right)$, since $t$ has an inverse in $I\left(y_{1}, y_{0}\right)$ represented by some element of $E(X)$. But the $E(X)$-orbit of $e \in I\left(y_{0}, y_{0}\right)$ is $I$, by definition. So the $E(X)$-orbit of $t$ is $I$, since $E(X)$ is a semigroup.

(d) The action map

$$
G \times I \rightarrow I, \quad(g, t) \rightarrow g \circ t,
$$

is continuous. A fibrewise metric for $\beta$ is given by

$$
\rho_{I}\left(t_{1}, t_{2}\right)=\sup _{x \in X_{y_{0}}} \rho\left(t_{1}(x), t_{2}(x)\right),
$$

if $\beta\left(t_{1}\right)=\beta\left(t_{2}\right)$. Continuity of $\rho$ follows from the remark in the proof of lemma 1.3 comparing $\rho$ and $d$.

Corresponding to the action of $E(X)$ on $I$ we have a semigroup homomorphism $E(X) \rightarrow I^{I}$. It is continuous with respect to the topology of pointwise convergence 
on $I$, by (c). So its image $E$ is compact and $G$ is dense in $E$, since it is dense in $E(X)$. This implies $E(I)=E$ by definition of the Ellis semigroup.

$I$ is a minimal $G$-space, since the $E$-orbit of every point of $I$ is all of $I$.

(e) We have first to explain the notation $I \times_{K} X_{y_{0}}$. The compact topological group $K$ has a right continuous action on $I$ by (b), and a left continuous action on $X_{y_{0}}$ by definition, hence a diagonal action on $I \times X_{y_{0}}$. Its orbit space is denoted $I \times{ }_{K} X_{y_{0}}$. The orbit of $(t, x) \in I \times X_{y_{0}}$ is denoted $[t, x]$. We have

$$
[t, x]=\left[t k^{-1}, k x\right]
$$

for $k \in K$.

The map $I \times X_{y_{0}} \rightarrow X$ is surjective, since for every $y \in Y$ there is an element $t \in I\left(y_{0}, y\right)$ which is an invertible isometry $X_{y_{0}} \rightarrow X_{y}$, in particular surjective. If $t_{1} x_{1}=t_{2} x_{2}$ for two elements $t_{1}, t_{2}$ in $I$ and two points $x_{1}, x_{2}$ in $X_{y_{0}}$, we have

$$
\pi\left(t_{1} x_{1}\right)=\pi\left(t_{2} x_{2}\right)
$$

so $t_{1}$ and $t_{2}$ map $X_{y_{0}}$ to the same fibre of $\pi, X_{y}$ say. Then $t_{2}^{-1} \circ t_{1}=k$ is in $I\left(y_{0}, y_{0}\right)$ and we have

$$
k x_{1}=t_{2}^{-1} t_{1} x_{1}=x_{2},
$$

hence

$$
\left[t_{1}, x_{1}\right]=\left[t_{2} \cdot k, x_{1}\right]=\left[t_{2}, k x_{1}\right]=\left[t_{2}, x_{2}\right] .
$$

So the map $I \times_{K} X_{y_{0}} \rightarrow X$ is a bijective continuous map of compact Hausdorff spaces, hence a homeomorphism.

(f) Right translations in $E(X)$ are continuous. This gives continuity of

$$
I\left(y_{0}, Y\right) \rightarrow I\left(y_{1}, Y\right), \quad s \mapsto s \circ,
$$

with respect to the topology of pointwise convergence. The same argument with $t^{-1}$ gives the continuous inverse mapping.

Remark 1.5. In the course of the proof we have obtained the following two statements: for every two points $y_{0}, y_{1}$ of $Y$ the set $I\left(y_{0}, y_{1}\right)$ is not empty; every element of $I\left(y_{0}, y_{1}\right)$ has an inverse in $I\left(y_{1}, y_{0}\right)$. We can consider $Y$ as the set of objects of a category $\mathscr{I}$ whose set of morphisms from $y_{0}$ to $y_{1}$ is $I\left(y_{0}, y_{1}\right)$. In the language of category theory the above two statements are equivalent with the following one: $\mathscr{I}$ is a connected groupoid. Is it a topological groupoid in a natural way? If Mor $(\mathscr{F})$ is given the identification topology from

$$
E(X) \times Y \rightarrow \operatorname{Mor}(\mathscr{I}), \quad(f, y) \rightarrow f_{y}: X_{y} \rightarrow X_{\pi_{*}(t) y},
$$

then the composition map $\mathscr{I} \times_{Y} \mathscr{I} \rightarrow \mathscr{I}$ is not continuous, in general, because composition in $E(X)$ is not continuous, in general. We only have continuity of

$$
I\left(y_{1}, Y\right) \times I\left(y_{0}, y_{1}\right) \rightarrow I\left(y_{0}, Y\right),
$$

as a consequence of lemma 1.3.

Remark 1.6. The starting point for our result 1.4 was [10]. Knapp's result is formulated in the language of function algebras; ours is more general, since we do not suppose $Y$ to be distal. 
Remark 1.7. One could also define a principal fibre bundle $B$ associated to $\pi: X \rightarrow Y$ by defining

$$
B=\bigcup_{y \in Y}\left\{\text { isometries } M \rightarrow X_{y}\right\}
$$

Its structure group is the full group of isometries of $M$. This definition has the advantage of avoiding the Ellis semigroup, but the disadvantage of depending on the fibrewise metric $\rho$ and having an unnecessarily big structure group. It gives a less naturally associated principal fibre bundle.

Remark 1.8. The 'principal fibre bundle' $I \rightarrow Y$ is not locally trivial, in general ([15, ex. 4.4, p. 390]). It is locally trivial if its structure group $K$ is a Lie group, by the slice theorem (c.f. [5]). Fortunately, we do not need local triviality, but only continuity of the quotient in denominator and numerator, as follows.

COROLlaRY 1.9. Let $\beta: I \rightarrow Y$ be the principal fibre bundle as in proposition 1.4. Let $K=I\left(y_{0}, y_{0}\right)$ be its structure group. Then the mapping

is continuous.

$$
\begin{gathered}
I \times \times_{Y} I \rightarrow K \\
\left(t_{1}, t_{2}\right) \mapsto t_{1}^{-1} \circ t_{2}
\end{gathered}
$$

Proof. The elements $t_{1}$ and $t_{2}$ belong to the same fibre $I\left(y_{0}, y\right)$ of $I$. So $t_{1}^{-1}$ exists and is an element of $I\left(y, y_{0}\right)$ and the composite map $t_{1}^{-1} \circ t_{2}$ is in $K=I\left(y_{0}, y_{0}\right)$. So the above map is well defined. To prove its continuity, look at the map

$$
\begin{aligned}
I \times K & \rightarrow I \times_{Y} I=\{(t, s): \beta(t)=\beta(s)\}, \\
(t, k) & \mapsto(t, t \circ k) .
\end{aligned}
$$

By 1.4(b) it is a continuous bijection of compact spaces, hence a homeomorphism. The map under consideration is the inverse homeomorphism followed by projection onto the $K$-component.

\section{The space of finite dimensional unitary representations}

In this section we shall prove our necessary condition for the existence of an effective distal action. To do this we shall need some notations and definitions.

Let $H$ be a locally compact $\sigma$-compact topological group. The space $\operatorname{Rep}_{n}(H)$ of continuous homomorphisms from $H$ into the group $U_{n}$ of complex unitary $n \times n$-matrices will be endowed with the topology of uniform convergence on compact subsets. The disjoint union

$$
\operatorname{Rep}_{\text {fin }}(H)=\bigcup_{n=0}^{\infty} \operatorname{Rep}_{n}(H)
$$

of open subsets $\operatorname{Rep}_{n}(H)$ will be called the space of continuous finite dimensional unitary representations of $H$; we call them just representations, for short. Since $H$ is $\sigma$-compact, $\operatorname{Rep}_{\text {fin }}(H)$ is a metrizable space. The group $U_{n}$ acts on $\operatorname{Rep}_{n}(H)$ by following a representation by conjugation with an element of $U_{n}$. Its orbit space

$$
\mathrm{Cl} \operatorname{Rep}_{n}(H)=\operatorname{Rep}_{n}(H) / U_{n}
$$

is called the space of equivalence classes of representations of dimension $n$. The 
equivalence class of $\phi \in \operatorname{Rep}_{n}(H)$ is denoted [ $\left.\phi\right]$. The disjoint union $\operatorname{Cl} \operatorname{Rep}_{\text {fin }}(H)$ of open subsets $\mathrm{Cl} \operatorname{Rep}_{n}(H)$ is called the space of equivalence classes of (continuous finite dimensional unitary) representations of $H$.

Let $\boldsymbol{A}$ be a locally compact topological group. Suppose we are given a continuous action of $A$ on $H$, such that every element $a \in A$ induces a topological automorphism of $H$. Then $A$ acts in a natural way continuously on the various spaces of representations of $H$, and $A \times U_{n}$ acts on $\operatorname{Rep}_{n}(H)$.

We are particularly interested in the following properties of a representation $\phi$.

LEMMA 2.1. For a representation $\phi$ of $H$ the following statements are equivalent:

(a) the closure $\overline{A \phi}$ of the $A$-orbit of $\phi$ in $\operatorname{Rep}_{\mathrm{fin}}(H)$ is a compact minimal A-space ;

(b) the closure $\overline{A[\phi]}$ of the $A$-orbit of $[\phi]$ in $\mathrm{Cl} \operatorname{Rep}_{\mathrm{fin}}(H)$ is a compact minimal A-space.

(c) The set

$$
\left\{\Psi \in \operatorname{Rep}_{\text {fin }}(H):[\Psi] \in \overline{A[\phi]}\right\}
$$

is a compact minimal $A \times U_{n}$-space contained in $\operatorname{Rep}_{n}(H)$, if $\phi$ has dimension $n$.

The same equivalences hold with 'compact minimal' replaced by 'compact minimal distal' in all statements. A representation with the above properties will be called $A$-minimal and A-distal, respectively.

Proof. Let us first prove the lemma for the 'compact minimal' statements. Since obviously (a) implies (b) and (b) implies (c), we have only to prove that (c) implies (a). Let $\Psi \in \overline{A \phi}$. We have to show that $\phi \in \overline{A \Psi}$. By (c) there is an element $u \in U_{n}$ such that

$$
I_{u} \circ \phi \in \overline{A \Psi}
$$

where $I_{u}$ is conjugation of $U_{n}$ by $u$. Since $\overline{A \Psi} \subset \overline{A \phi}$, this implies

$$
I_{u} \overline{A \Psi} \subset I_{u} \overline{A \phi} \subset \overline{A \Psi} .
$$

So $u$ belongs to the closed subsemigroup

$$
S=\left\{u \in U_{n}: I_{u} \overline{A \Psi} \subset \overline{A \Psi}\right\}
$$

of $U_{n}$. But every closed subsemigroup of a compact topological group is a group, hence $I_{u} \overline{A \Psi}=\overline{A \Psi}$, implying $\overline{A \Psi}=\overline{A \phi}$.

In order to prove the equivalences for 'compact minimal distal' we just invoke theorems on distal spaces. By a result of Ellis, the equivariant image of a compact distal $\boldsymbol{A}$-space is compact distal, proving (a) implies (b). It is straightforward that (b) implies (c); compactness follows from the fact that the map

$$
\operatorname{Rep}_{n}(H) \rightarrow \mathrm{Cl} \operatorname{Rep}_{n}(H)
$$

is the natural map of a space onto its orbit space by the action of a compact group, hence a proper map. If (c) holds, the space in (c) is a distal $\boldsymbol{A}$-space, hence every orbit closure is a minimal distal $A$-space, by another result of Ellis, implying (a).

The following lemmas reduce the question of minimality and distality to irreducible representations. 
LemMa 2.2. Let $\phi \in \operatorname{Rep}_{\text {fin }}(H)$ be $A$-minimal. Then we have

$$
\overline{\phi(H)}=\overline{\Psi(H)}
$$

for every $\Psi \in \overline{\boldsymbol{A} \phi}$. Hence if $\phi$ is irreducible, so is $\Psi$.

Proof. For every $a \in A$ we have

$$
(a \phi)(H)=\phi\left(a^{-1}(H)\right)=\phi(H) .
$$

So $\Psi(H) \subset \overline{\phi(H)}$ for every $\Psi \in \overline{A \phi}$. By minimality $\phi \in \bar{A} \Psi$, hence the inverse inclusion. The last sentence follows from the fact that $\phi$ is irreducible iff the identity is the only projection operator of the representation space commuting with $\phi(H)$, or equivalently, with $\overline{\phi(H)}$.

LeMmA 2.3. A representation $\phi \in \operatorname{Rep}_{\text {fin }}(H)$ is $A$-minimal (A-distal) iff all of its irreducible components are.

Proof. Let $\phi: H \rightarrow U_{n}$ be a representation. Let $P_{1}, \ldots, P_{r}$ be a maximal set of mutually orthogonal projection operators of $\mathbb{C}^{n}$ commuting with $\phi(H)$. The projections commute with $\Psi(H)$ for every $\Psi \in \overline{A \phi}$, since $\Psi(H) \subset \overline{\phi(H)}$. So restricting the representations $\Psi \in \overline{A \phi}=X$ to the image of $P_{i}$ defines a continuous $A$-map

$$
X \rightarrow \operatorname{Rep}_{\text {fin }}(H), \quad \Psi \mapsto \Psi_{i}
$$

Hence if $\phi$ is $A$-minimal ( $A$-distal), so are the $\phi_{i}$. The converse follows from the fact that the direct sum of representations defines a continuous $A$-map

$$
\operatorname{Rep}_{\text {fin }}(H) \times \operatorname{Rep}_{\text {fin }}(H) \rightarrow \operatorname{Rep}_{\text {fin }}(H) .
$$

Suppose $G$ is a locally compact $\sigma$-compact topological group and $N$ is a closed normal subgroup. The inner automorphisms of $G$ restricted to $N$ give an action of $G$ on $N$. So it makes sense to talk about $G$-distal representations of $N$.

Definition 2.4. A locally compact $\sigma$-compact topological group $G$ is called generalized maximally almost periodic, abbreviated to $G M A P$, if every closed normal subgroup $N$ of $G$ has a non-trivial $G$-distal representation. For a closed normal subgroup $N$ of $G$ define

$$
N^{(G)}=\bigcap_{\phi} \operatorname{ker} \phi
$$

where $\phi$ runs over all $G$-distal representations of $N$.

Let us call a transfinite sequence $\left\{N_{\beta}: 0 \leq \beta \leq \alpha\right\}, \alpha$ some ordinal, of closed normal subgroups $N_{\beta}$ of $G$ a GMAP-sequence (of length $\alpha$ ) if the following conditions hold:

(a) $N_{0}=G$

(b) $N_{\beta+1} \supset N_{\beta}^{(G)}$,

(c) for every limit ordinal $\beta=\sup \{\gamma: \gamma<\beta\}$ we have

$$
N_{\beta} \supset \bigcap_{\gamma<\beta} N_{\gamma}
$$

Obviously, $G$ is $G M A P$ iff there is an ordinal $\alpha$ and a $G M A P$-sequence $\left\{N_{\beta}: 0 \leq \beta \leq\right.$ $\alpha\}$ with $N_{\alpha}=\{e\}$. In fact, for every locally compact $\sigma$-compact topological group 
$G$ there is one fastest decreasing $G M A P$-sequence, defined by:

$$
N_{0}=G, \quad N_{\beta+1}=N_{\beta}^{(G)},
$$

and

$$
N_{\beta}=\bigcap N_{\gamma} \quad \text { if } \beta=\sup \{\gamma: \gamma<\beta\} .
$$

If $G$ is second countable, this sequence becomes constant from some countable ordinal $\alpha$ on. The terminology is justified by the following remark. $G$ is maximally almost periodic iff $G^{(G)}=\{e\}$, i.e. iff there is a $G M A P$-sequence of length 1 .

One of our main results is the following theorem.

THEOREM 2.5. Every locally compact $\sigma$-compact topological group admitting an effective distal action on some compact metric space is generalized maximally almost periodic.

Proof. Let $\boldsymbol{X}$ be a compact metric space with a minimal effective distal action of $G$. Let $\left\{X_{\beta}\right\}_{\beta \leq \alpha}$ be the transfinite series of extensions of Furstenberg's structure theorem (see 1.2). Let $N$ be a closed normal subgroup of $G$. Let $\beta$ be the least ordinal such that $N$ acts non-trivially on $X_{\beta}$. The ordinal $\beta$ is not a limit ordinal, because if $N$ acts trivially on $X_{\gamma}, \gamma<\beta$, it also acts trivially on

$$
X_{\beta}=\operatorname{inv} \lim _{\gamma<\beta} X_{\gamma} .
$$

So $\beta=\gamma+1$ and we have an isometric extension $\pi: X_{\gamma+1} \rightarrow X_{\gamma}$ of distal $G$-spaces such that $N$ acts trivially on $X_{\gamma}$, but non-trivially on $X_{\gamma+1}$. By proposition 1.4 there is a principal $G$-fibre bundle

$$
I \rightarrow Y:=X_{\gamma}
$$

with compact structure group $K$. The left action of $G$ on $I$ is distal. It commutes with the right action of $K$ on $I$. Let $n_{0} \in N$ and $x_{0} \in X_{y+1}$ be such that $n_{0} x_{0} \neq x_{0}$. Define $y_{0}=\pi\left(x_{0}\right)$. Let

$$
\sigma: N \rightarrow I\left(y_{0}, y_{0}\right)=K
$$

be the continuous homomorphism given by restricting the maps of $N$ to the fibre $\pi^{-1}\left(y_{0}\right)$. We have $\sigma\left(n_{0}\right) \neq e$. Let $\rho: K \rightarrow U_{n}$ be a representation of $K$ such that

$$
\rho \circ \sigma\left(n_{0}\right) \neq e \text {. }
$$

We show that $\phi:=\rho \circ \sigma$ is a $G$-distal representation of $N$ thus proving the theorem. We do this by defining a continuous $G$-map

$$
\Phi: I \rightarrow \operatorname{Rep}_{n}(N) \text { such that } \Phi\left(y_{0}\right)=\phi .
$$

Using the notations of proposition 1.4 , define $\Phi(t) \in \operatorname{Rep}_{n}(N)$ by

$$
\Phi(t)(a)=\phi\left(t^{-1} a t\right) \quad \text { for } t \in I=I\left(y_{0}, y\right) .
$$

The map

$$
I \times N \rightarrow K, \quad(t, a) \rightarrow t^{-1} a t=t^{-1}(a t),
$$

is continuous, since by $1.4(\mathrm{~d})$ the map

$$
N \times I \rightarrow I, \quad(a, t) \rightarrow a t,
$$


is continuous and by 1.9 the map

$$
I \times_{Y} I \rightarrow K, \quad\left(t_{1}, t_{2}\right) \rightarrow t_{1}^{-1} t_{2},
$$

is continuous. So $\Phi$ is continuous in both variables, hence gives a continuous map $\Phi: I \rightarrow \operatorname{Rep}_{n}(N)$, which is a $G$-map by a straightforward computation.

This is the only place where we make an application of the principal bundle $I \rightarrow Y$ with $G$-action constructed in $\S 1$. We do not make full use of the bundle structure, we just look at the representation classes of the ineffective kernel $N$ of $Y$ given by the bundle.

For arbitrary $G$ there is no effective distal action of $G$, but there is a smallest ineffective kernel for distal actions of $G$, as follows.

LEMMA 2.6. Let $G$ be a locally compact second countable topological group and let $N$ be the intersection of the ineffective kernels of all distal actions of $G$. Then $G$ admits a minimal distal action on a compact metric space, which has a point with isotropy group precisely $N$.

Proof. We may assume $N=\{e\}$. For every distal $G$-space $X$ and every point $x \in X$ the complement of the isotropy group

$$
G_{x}=\{g \in G: g x=x\}
$$

is an open subset of $G$. The union of these $G \backslash G_{x}$ is $G \backslash\{e\}$. Since $G$ is second countable, a countable subcover of $G \backslash\{e\}$ exists; hence there is a countable set $\left(X_{i}, x_{i}\right)$ of pointed distal $G$-spaces such that

$$
\bigcap G_{x_{i}}=\{e\} .
$$

The compact metric space $Y=\prod X_{i}$ is distal and the closure of the $G$-orbit of $y=\left(x_{i}\right)_{i} \in Y$ has the required properties.

Theorem 2.5 implies under these circumstances the following corollary.

COROLLARY 2.7. With the hypotheses and notations of the lemma, there is a countable ordinal $\alpha$ and a GMAP-sequence $\left\{N_{\beta}: 0 \leq \beta \leq \alpha\right\}$ for $G$ such that $N_{\alpha} \subset N$.

Proof. By theorem 2.5 and the remarks following definition 2.4 there is a countable ordinal $\alpha$ and a $G M A P$-sequence $\left\{M_{\beta}: 0 \leq \beta \leq \alpha\right\}$ for $G / N$ such that $M_{\alpha}=\{e\}$. The sequence $\left\{N_{\beta}: 0 \leq \beta \leq \alpha\right\}$ of inverse images of $M_{\beta}$ under the natural map $G \rightarrow G / N$ is a $G M A P$-sequence for $G$.

\section{Applications for connected groups}

Let us suppose all locally compact topological groups occurring are second countable. In this section we first give some general properties of GMAP-groups, then we show that for almost connected $G$ the properties $G M A P$, polynomial growth and existence of an effective distal action are equivalent. In the next section we discuss the example of the rational $a x+b$-group.

Proposition 3.1. Let $G$ be GMAP. Then for every element $g \neq e$ of $G$ the closure of the conjugacy class of $g$ does not contain the identity element $e$. 
Proof. Suppose there is an element $g \neq e$ of $G$ and a net $\left\{g_{i}\right\}$ in $G$ such that $g_{i} g g_{i}^{-1}$ converges to $e$. Let $N$ be the smallest closed normal subgroup of $G$ containing $g$. There is a $G$-distal representation $\phi$ of $N$ such that $g$ is not in the kernel of $\phi$. Since $\overline{G \phi}$ is compact we may assume that the net $g_{i} \phi$ converges to some representation $\Psi$ of $N$. Then $\phi(g)=\left(g_{i} \phi\right)\left(g_{i} g g_{i}^{-1}\right)$ converges to $\Psi(e)$, hence $\phi(g)=\Psi(e)$, a contradiction. Note that we have made use of the compactness of $\overline{G \phi}$ only.

This proposition implies, by [13], [2], one of the main results of [12].

COROLlARY 3.2. Suppose $G$ is an almost connected locally compact second countable topological group. If $G$ acts effectively and distally on some compact space then $G$ has polynomial growth.

We shall prove the converse below. We now show unimodularity of $G M A P$-groups.

LeMMA 3.3. Let $H$ be a locally compact topological group. Let the group $A$ act on $H$ by automorphisms. If the A-compact representations of $H$ separate the points of $H$, then every left Haar measure of $H$ is a right Haar measure and is $A$-invariant.

Proof. The inner automorphisms of $H$ and the automorphisms of $H$ induced by elements of $A$ together give an action of $A \ltimes H$ on $H$, for which there is a separating family of $A \ltimes H$-compact representations by lemma 2.1 . So it suffices to prove the invariance of a, say left, Haar measure $\mu$ with respect to this group of automorphisms, which we call $A$ again.

Let $U$ be a compact neighbourhood of $e$ in $H$. Suppose there is an element $a \in A$ with $\mu(a U)>\mu(U)$. Let $V$ be a (small) neighbourhood of $e$ in $H$. Then there is an $n \in \mathbb{N}$ such that $\mu\left(a^{n} V\right)>\mu(U)$, hence $a^{n} V \not \subset U$. Let $n \in \mathbb{N}$ be minimal with the property $a^{n} V \not \subset U$. Then $a^{n} V \subset a U$. Let

$$
L=a U \backslash \stackrel{\circ}{ }
$$

If we let $V$ run through a neighbourhood base of $e$, we obtain a net $v_{i}$ of elements converging to $e$ and $n_{i} \in \mathbb{N}$ with

$$
a^{n_{i}} v_{i}=l_{i} \in L .
$$

We may assume that the net $l_{i}$ converges to some element $l$ in the compact set $L$. Now let $\phi$ be an $A$-compact representation in $\operatorname{Rep}_{\text {fin }}(H)$. We may assume that $a^{-n_{i}} \phi$ converges to some $\Psi \in \operatorname{Rep}_{\text {fin }}(H)$. Then

$$
\phi(l)=\lim \phi\left(a^{n_{i}} v_{i}\right)=\lim \left(a^{-n_{i}} \phi\right)\left(v_{i}\right)=\Psi(e) .
$$

So $l$ is contained in the intersection of the kernels of all $A$-compact representations of $H$, hence $l=e$, a contradiction.

COROllary 3.4. Every GMAP-group is unimodular.

Proof. By induction on the length $\alpha$ of a $G M A P$-sequence $\left\{N_{\beta}: 0 \leq \beta \leq \alpha\right\}$ for our group $G$ with $N_{\alpha}=\{e\}$.

If $G / N_{\alpha}$ is unimodular and $G$ acts in a unimodular fashion on $N_{\alpha} / N_{\alpha+1}$, then $G / N_{\alpha+1}$ is unimodular. This is the inductive step for $\alpha \rightarrow \alpha+1$. If $\alpha$ is a limit ordinal

$$
\alpha=\sup \{\beta: \beta<\alpha\} \quad \text { and } \bigcap_{\beta<\alpha} N_{\beta}=N_{\alpha}=\{e\}
$$


suppose there is an element $g \in G$ that does not leave a left Haar measure of $G$ invariant. Let $V$ be a compact symmetric neighbourhood of $e$ in $G$ containing $g$. Set

$$
L=V^{3} \backslash \stackrel{\circ}{V}
$$

The descending sequence $L \cap N_{\beta}$ of compact subsets of $L$ has zero intersection, hence there is a $\beta<\alpha$ with

hence

$$
N_{\beta} \cap L=\varnothing,
$$

So

$$
N_{\beta} \cap V^{3} \subset N_{\beta} \cap V .
$$

$$
g\left(N_{\beta} \cap V\right) g^{-1} \subset N_{\beta} \cap V^{3} \subset N_{\beta} \cap V,
$$

and the same for $g^{-1}$. Hence $N_{\beta} \cap V$ is a compact $g$-invariant neighbourhood of $e$ in $N_{\beta}$, so $g$ leaves every Haar measure of $N_{\beta}$ invariant. By the inductive hypothesis $G / N_{\beta}$ is unimodular, hence $g$ leaves every Haar measure of $G$ invariant, a contradiction.

Starting from this corollary one can obtain a different proof of corollary 3.2 , basically by making use of the facts that the real $a x+b$-group and a similar 3-dimensional group are not unimodular and every connected Lie group that is not polynomially growing contains one of these groups, locally.

The converse of corollary 3.2 is true, too. Since this fact is not proved in [12], not even claimed, we shall give a proof.

THEOREM 3.5. An almost connected locally compact second countable topological group admits an effective distal action iff it is polynomially growing.

The proof of the theorem depends on the following two results, the first of which may be of independent interest (cf. Auslander's semisimple splitting [3]).

THEOREM 3.6. Every polynomially growing connected Lie group $G$ can be embedded as a closed normal subgroup into a connected Lie group, which has a closed connected normal nilpotent subgroup with compact factor group.

The 'almost nilpotent' extension of $G$ we construct is actually a semidirect product of $G$ with a torus.

Proof. Let $G$ be a connected Lie group of polynomial growth. The adjoint action of $G$ on its Lie algebra $g$ defines a homomorphism

$$
\text { Ad: } G \rightarrow G l(g) \text {. }
$$

Let $A$ be the algebraic hull of $\operatorname{Ad}(G)$ in $G l(g)$. The group $G$ acts on itself by inner automorphisms, thus giving a homomorphism

$$
\text { Ad }(G) \rightarrow \text { Aut }(G) \text {. }
$$

The first and main step of the proof is to show that this homomorphism extends to an analytic homomorphism $A \rightarrow$ Aut $(G)$. Let $\hat{G}$ be the simply connected covering group of $G$, let

$$
\exp : g \rightarrow \hat{G}
$$

be its exponential map. Let $D$ be the kernel of $\hat{G} \rightarrow G$. Identifying Aut $(\hat{G})$ with 
Aut ( $g$ ) we have to show that $A$ leaves $D$ invariant. Let $K$ be the maximal compact connected subgroup of the centre of $G$, let $k$ be its Lie algebra and let $\hat{K}$ be the corresponding subgroup of $\hat{G}$, a closed central vector subgroup of $\hat{G}$. Since $G$ has polynomial growth, the adjoint action of $G$ on $g$ is distal $([13])$ and by $[2$, lemma 2.6] $A$ leaves $D \cdot \hat{K}$ invariant. Since $\operatorname{Ad}(G)$ is connected and distal, its algebraic hull $A$ is connected (and distal) by $[1,2.7]$. So for every $d \in D$ the continuous homomorphism

$$
\phi_{d}: A \rightarrow D \cdot \hat{K}, \quad \phi_{d}(f)=f(d) \cdot d^{-1},
$$

takes values in $\hat{K}$. By construction, $\phi_{d}$ vanishes on $\operatorname{Ad}(G)$. But $A / \operatorname{Ad}(G)^{-}$is compact by [1, theorem 3], so the image of $\phi_{d}$ is a compact subgroup of the vector group $\hat{K}$, hence trivial. That is, $A$ fixes $D$, hence $A$ acts on $G$.

Now $A=U \ltimes L$, where $U$ is the normal subgroup of unipotent elements of $A$ and $L$ is a maximal compact subgroup of $A([1], 2.5])$. Let $Z$ be the connected component of the centre of $L \subset$ Aut $(G)$ and define

$$
H=G \times Z \text {. }
$$

We claim that $H$ is the desired supergroup of $G$. To see this first look at $H_{1}=A \times Z$, where $Z \subset A$ acts on $A$ by conjugation. The radical of $H_{1}$ is $U \cdot Z \times Z$ and

$$
H_{1} / \operatorname{rad} H_{1} \cong L / Z
$$

is compact semisimple. Let

$$
D=\left\{\left(z, z^{-1}\right): z \in Z\right\} \subset Z \times Z \subset A \times Z=H_{1} .
$$

Then $D$ is a compact connected central subgroup of $H_{1}$, hence $U \cdot D$ is a closed connected nilpotent normal subgroup of $H_{1}$.

Let us come back to $H=G \times Z$. The adjoint representation of $G$ together with the identity on $Z$ gives a homomorphism

$$
\phi: H=G \rtimes Z \rightarrow A \times Z=H_{1} .
$$

Let $B$ be its kernel. $B$ is the centre of $G$. So the algebraic hull $A$ of $\operatorname{Ad} G$ acts trivially on its Lie algebra $b$, hence on its connected component $B_{0}$. Furthermore $A$ leaves the characteristic subgroup $B$ of $G$ invariant, so $A$ acts on the discrete group $B / B_{0}$. This action is trivial, since $A$ is connected. So

$$
\{e\} \subset B_{0} \subset B
$$

is an $\boldsymbol{A}$-invariant filtration such that $\boldsymbol{A}$ acts trivially on its quotients. This implies that $B_{0}$ is central in $H$ and $B / B_{0}$ is central in $H / B_{0}$. In particular $M=\phi^{-1}(U \cdot D)$ is a nilpotent closed normal subgroup of $H$. We claim that $H / M_{0}$ is compact.

Let us write $Z_{2}, Z_{3}$ for $\{e\} \times Z$ in $H$ and $H_{1}$ respectively. Note that $Z_{3}$ normalizes $U$ and $D$, hence $Z_{2}$ normalizes $M$. The subgroup $M \cdot Z_{2}$ of $H$ is the inverse image of

$$
U \cdot Z \rtimes Z_{3}=\operatorname{rad} H_{1} .
$$

So $\phi$ induces an injective homomorphism

$$
H / M Z_{2} \rightarrow H_{1} / \operatorname{rad} H_{1} \cong L / Z
$$

whose image has $L / Z$ as algebraic hull, since the algebraic hull of $\operatorname{Ad} G$ is $A$. So 
the connected Lie group $H / M Z_{2}$ has an injective homomorphism into a compact semisimple Lie group with dense image, hence $H / M Z_{2}$ is compact semisimple itself (see lemma 3.7 below). It follows that $H$ modulo the connected component $M_{0} \cdot Z_{2}$ of $M Z_{2}$ is locally isomorphic to a compact semisimple Lie group, hence $H / M_{0} Z_{2}$ is compact semisimple.

LEMMA 3.7. Let $H$ be a connected Lie group and let $G$ be a compact connected semisimple Lie group. Suppose $\phi: H \rightarrow G$ is an injective Lie group homomorphism with dense image. Then $\phi$ is an analytic isomorphism $H \rightarrow G$.

Proof. The closure of the image of the radical of $H$ is a closed connected solvable normal subgroup of $G$, hence trivial. So $H$ is semisimple. The Killing form of $g$ restricted to $h$ via $\phi$ gives a negative definite invariant symmetric bilinear form on $h$, so $h$ is the Lie algebra of a compact Lie group ([9, chap. XIII, Theorem 1.1]). Since $h$ is semisimple, $H$ must be compact. The result follows.

LEMMA 3.8. Every nilpotent connected Lie group has an effective distal action.

Proof. The proof is similar to that of Moore and Zimmer ([12, p. 183f.]) for the measure theoretic case, but simpler. Let $N=\tilde{N} / D$, where $\tilde{N}$ is a simply connected nilpotent Lie group and $D$ a discrete normal, hence central, subgroup of $\tilde{N}$. We consider $\tilde{N}$ as an algebraic group with its unique structure as a unipotent real algebraic group. Let $M$ be the smallest algebraic subgroup of $\tilde{N}$ containing $D$. If we denote by exp the exponential map $n \rightarrow \tilde{N}$ and by log its inverse, we have

$$
M=\exp m,
$$

where $m$ is the smallest vector space containing $\log D$. Since $D$ is free abelian, it has a basis, $\left\{e_{i}\right\}_{i}$ say. Let $D_{i}$ be the group generated by the $\left\{e_{j}: j \neq i\right\}$ and let $M_{i}$ be the smallest connected Lie subgroup of $\tilde{N}$ containing $D_{i}$. Then by lemma 2.6 it suffices to show, that $\tilde{N} / D \cdot M_{i}$ has an effective distal action for every $i$. So we may assume that rank $D \leq 1$.

If $D=\{e\}$ consider $\tilde{N}=N$ as a subgroup of the group $N_{n}$ of upper triangular real $n \times n$-matrices with ones on the diagonal. Let $\Gamma_{p}$ be the subgroup of $N_{n}$ of matrices with integer entries whose off-diagonal entries are divisible by $p$. The group $\Gamma_{p}$ is a discrete subgroup of $N_{n}$, and $N_{n}$ acts distally on the compact metric space $N_{n} / \Gamma_{p}$ [4]. The ineffective kernel is the intersection of $\Gamma_{p}$ with the centre of $N_{n}$. Since the intersection of all $\Gamma_{p}$, for $p$ prime, is $\{e\}, N_{n}$ has an effective distal action, by lemma 2.6, hence $N$ has an effective distal action.

If rank $D=1$, again consider $\tilde{N}$ as a subgroup of $N_{n}$. Let $g$ be a generator of $D$. There is a basis

$$
e_{n}, e_{n-1}, \ldots, e_{1} \quad \text { of } \mathbb{R}^{n}
$$

with respect to which $\tilde{N}$ is still triangular with ones on the diagonal and such that for every $j \in\{1, \ldots, n\}$ we have

$$
(g-I d) e_{j} \text { is zero or is some } e_{i} \text { with } i<j .
$$

It follows that we may assume that $\tilde{N}$ is a subgroup of $N_{n}$ and the elements of $D$ have integer entries. 
The set $D \cdot \Gamma_{p}$ of products of elements of $D$ and of $\Gamma_{p}$ is a group, since $D$ normalizes $\Gamma_{p}$. The group $D \cdot \Gamma_{p}$ contains $\Gamma_{p}$ as a subgroup of finite index, so $N_{n} / \Gamma_{p} \cdot D$ is a distal $N_{n}$-space and in particular a distal $\tilde{N}$-space. Let $X_{p}$ be the closure of the $\tilde{N}$-orbit of $x_{0}=\Gamma_{p} \cdot D$ in $N_{n} / \Gamma_{\mathrm{p}} \cdot D$. Since the normal subgroup $D$ of $\tilde{N}$ fixes $x_{0}$, it acts trivially on $X_{p}$, so $X_{p}$ is a distal $N$-space. The ineffective kernel of $N x_{0}$ and hence of $X_{p}$ is

$$
\Lambda_{p}=Z(N) \cap D \Gamma_{p} / D .
$$

Since the intersection of the $\Lambda_{p}$ 's is trivial, $N$ has an effective distal action in this case, too.

We are now ready to give a proof of theorem 3.5. We have to show that every polynomially growing locally compact second countable topological group $G$ admits an effective distal action. In view of lemma 2.6 it suffices to give a proof for Lie groups, since our group is an inverse limit of polynomially growing Lie groups with finite component group. Furthermore we may assume that $G$ is connected, because if $X_{0}$ is an effective distal $G_{0}$-space, then

$$
\text { ind }_{G_{0}}^{G} X_{0}=\left(X_{0} \times G\right) / G_{0}
$$

is an effective distal $G$-space. By theorem 3.6 we may now assume that $G$ has a closed connected normal nilpotent subgroup $N$ with compact factor group $G / N$, since obviously a subgroup of a group admitting an effective distal action itself does. Finally we may assume that $N=G$ is nilpotent, because if $X_{0}$ is an effective distal $N$-space, then ind ${ }_{N}^{G} X_{0}$ is a compact effective distal $G$-space, since $G / N$ is compact. For nilpotent $N$ lemma 3.8 proves our claim.

\section{Applications for discrete groups}

For discrete groups proposition 3.1 and corollary 3.4 give no information, but theorem 2.5 does; e.g. we have the following result:

COROLlaRY 4.1. Let $G$ be a finitely generated discrete group. Let $M$ be the intersection of all (normal) subgroups of $G$ of finite index. If $M$ is of finite index in $G$, then $M$ is the smallest ineffective kernel of distal actions of $G$.

Proof. We make use of the results of Malcev [11] that a finitely generated linear group over the complex numbers is residually finite, i.e. the intersection of all (normal) subgroups of finite index is $\{e\}$. Hence if $H$ is a subgroup of finite index of our group $G$ and $\phi$ is a finite dimensional representation of $H$, we have $\operatorname{ker} \phi \supset M$, since $H$ is finitely generated. Now suppose $M$ is of finite index in $G$. Then for every $G M A P$-sequence $\left\{N_{\beta}\right\}$ of $G$ it follows that $N_{\beta} \supset M$. Hence $M$ is contained in the kernel of any distal action of $G$. On the other hand, the finite group $G / M$ has a free isometric action.

Remark 4.2. E.g. Higman's group [8]

$$
G=\left\langle a, b, c, d ; a b a^{-1}=b^{2}, b c b^{-1}=c^{2}, c d c^{-1}=d^{2}, d a d^{-1}=a^{2}\right\rangle
$$

is finitely presented, but the only subgroup of $G$ of finite index is $G$ itself, so the only distal actions of $G$ are the trivial ones. 
The situation for non finitely generated groups is entirely different. E.g. the additive group $\mathbb{Q}$ of rational numbers has no proper subgroup of finite index, but, regarded as a discrete group, it has a faithful 1-dimensional representation, hence a free isometric action. More generally, every abelian locally compact second countable group has a countable separating family of characters, by Pontryagin duality, hence an isometric free action. On the other hand, not every metabelian discrete group admits an effective distal action, as the following example shows.

EXAMPLE 4.3. The discrete group $B$ of affine maps

$$
\mathbb{Q} \rightarrow \mathbb{Q}, \quad x \mapsto a x+b
$$

of the rational line, $b \in \mathbb{Q}, a \in \mathbb{Q}^{*}$, has no effective distal action. In fact, the smallest kernel for distal actions is the subgroup $N$ of translations, $N=\{a=1\}$.

Proof. Let $M$ be the intersection of the ineffective kernels of all distal actions of $B$. We have $M \subset N$, since $B / N \simeq \mathbb{Q}^{*}$ admits an injective homomorphism into $U_{1}$ defining a free isometric action on $U_{1}$. To prove the converse it suffices by corollary 2.7 to show that the trivial representation of $N$ is the only $B$-distal representation of $N$. Let $\phi \in \operatorname{Rep}_{n}(N)$ be a $B$-distal $n$-dimensional representation. By lemma 2.3 we may assume that $\phi$ is irreducible, so $n=1$ and $\phi$ is an element of the dual group $\hat{N}$ of $N \simeq \mathbb{Q}$. For an element $g$ of $B$ with

$$
g(x)=\frac{1}{n} x+b
$$

we have

$$
(g \circ \phi)(y)=\phi(n y), \quad y \in \mathbb{Q},
$$

so all powers $\phi^{n}, n \neq 0, n \in \mathbb{Z}$, are in the $B$-orbit of $\phi$. Since $\hat{\mathbb{Q}}$ is a compact topological group, it follows that the closure $X$ of the $B$-orbit contains the trivial representation $\phi^{0}$. But if $X$ is a distal $B$-space, it is minimal, since it is an orbit closure, hence

$$
\phi \in\left(B \phi^{0}\right)^{-}=\phi^{0},
$$

so $\phi$ is the trivial representation.

The examples 4.6 below tell us that big subgroups of $B$ admit effective distal actions. We shall make use of the following lemma, which may be considered as a partial converse of theorem 2.5 .

Lemma 4.4. Suppose $H$ and $N$ are locally compact groups and suppose $H$ acts continuously on $N$ by automorphisms. Let $G$ be the split extension $G=H \ltimes N$. For every $H$-distal representation $\phi$ of $N$ there is a distal action $\Phi$ of $G$ with

$$
\text { Ineff }(\Phi) \cap N \subset \operatorname{ker} \phi .
$$

Here Ineff $(\Phi)$ denotes the ineffective kernel of the action $\Phi$. The lemma implies the following corollary.

COROllary 4.5. If $H$ admits an effective distal action and $N$ has a separating family of $H$-distal representations then $G=H \ltimes N$ admits an effective distal action. 
Proof of lemma. Let us denote the action of $H$ on $N$ by

$$
(h, n) \mapsto{ }^{h} n .
$$

The topological group $G$ is the product space $N \times H$ with the multiplication

$$
\left(n_{1}, h_{1}\right) \cdot\left(n_{2}, h_{2}\right):=\left(n_{1} \cdot{ }^{h_{1}} n_{2}, h_{1} h_{2}\right) .
$$

We identify $n \in N$ with $(n, e) \in G$. We define an action of $G$ on $U_{m} \times \operatorname{Rep}_{m}(N)$ by

$$
\begin{aligned}
(n, h)(u, \phi) & =(((n, h) \circ \phi)(n) \cdot u,(n, h) \circ \phi) \\
& =\left(\phi\left({ }^{h-1} n\right) \cdot u,(n, h) \circ \phi\right) .
\end{aligned}
$$

Here, as usually, $G$ acts on $\operatorname{Rep}_{m}(N)$ by

$$
\begin{aligned}
((n, h) \circ \phi)(x) & =\phi\left((n, h)^{-1} \cdot x \cdot(n, h)\right) \\
& =\phi\left(\left(^{h^{-1}}\left(n^{-1} \cdot x \cdot n\right)\right) .\right.
\end{aligned}
$$

It is straightforward to check that this defines an action of $G$ on

$$
X=U_{m} \times \operatorname{Rep}_{m}(H),
$$

which thus becomes an isometric extension of the (non-compact) $G$-space $\operatorname{Rep}_{m}(H)$. Restricting to the part of $X$ over the closure of the orbit of $\phi$ gives the desired compact distal $G$-space.

We are now ready for the examples. Let $\omega_{p}$ be the $p$-adic valuation of $\mathbb{Q}^{*}$, defined by

$$
\omega_{p}\left(p^{n} \frac{a}{b}\right)=n,
$$

if $a$ and $b$ are integers not divisible by $p$.

ExAmples 4.6. Again let $B$ be the group of affine maps of the rational line. The subgroup $C$ of $B$ of maps

$$
\mathbb{Q} \rightarrow \mathbb{Q}, \quad x \mapsto a x+b \quad \text { with } \omega_{p}(a)=0
$$

admits an effective 2-distal action, but is not maximally almost periodic.

Proof. The group $B$ is the split extension $\mathbb{Q}^{*} \propto \mathbb{Q}$ with respect to the action

Set

$$
\mathbb{Q}^{*} \times \mathbb{Q} \rightarrow \mathbb{Q}, \quad(a, b) \mapsto a \cdot b .
$$

$$
\boldsymbol{A}=\operatorname{ker} \omega_{p} \subset \mathbb{Q}^{*} .
$$

In order to show that $C=A \times \mathbb{Q}$ admits an effective distal action, it suffices by corollary 4.5 to show that $\mathbb{Q}$ has a separating family of $\boldsymbol{A}$-distal characters. We claim that a character $\phi: \mathbb{Q} \rightarrow S^{1}$ is $A$-distal, if its values are in the group $W$ of elements of $S^{1}$ whose order is a power of $p$. These characters certainly form a separating family. For every integer $a \in A$ the map $x \rightarrow x^{a}$ is an automorphism of the finite group

$$
W(n)=\left\{x \in \mathbb{C}: x^{p^{n}}=e\right\}
$$

of $p^{n}$ 'th roots of unity. The map

$$
A \cap \mathbb{Z} \rightarrow \text { Aut }(W(n))
$$

extends to a homomorphism $A \rightarrow \operatorname{Aut}(W(n))$, thus giving an action of $A$ on each $W(n)$, and hence on $W$, also denoted $(a, x) \mapsto x^{a}$. 
Now let $\phi$ be a character $\phi: \mathbb{Q} \rightarrow W$. We have $\phi\left(p^{m}\right)=1$ for some power $p^{m}$ of p. It follows that

$$
\phi\left(p^{m-n}\right) \in W(n) .
$$

The character is uniquely determined by the values of $p^{k}, k \in \mathbb{Z}$, since

$$
\phi\left(p^{k} \cdot a\right)=\left(\phi\left(p^{k}\right)\right)^{a} \quad \text { for } a \in A \text {. }
$$

It follows that the group $D_{m}$ of characters

$$
\Psi: \mathbb{Q} \rightarrow W \quad \text { with } \Psi\left(p^{m}\right)=1
$$

is isomorphic to the inverse $\operatorname{limit}_{\hbar} W(n)$ of the system of groups $W(n)$ with maps

$$
W(n+1) \rightarrow W(n), \quad z \mapsto z^{p} .
$$

The group $A$ acts isometrically on every $W(n)$ hence on their inverse limit. In particular $\phi$ is contained in a compact isometric $A$-subspace of $\operatorname{Rep}_{1}(\mathbb{Q})$, and is $a$ fortiori an $\boldsymbol{A}$-distal representation.

It is easy to see that $C$ is not maximally almost periodic. In fact $\mathbb{Q}$ is the intersection of all kernels of (finite dimensional unitary) representations of $C$. To see this, let $\phi \in \operatorname{Rep}_{n}(C)$. Let $K$ be the closure of the image of $\phi$. The connected component $K_{0}$ of $K$ is a compact connected solvable Lie group, hence abelian, of finite index in $K$. Since the only subgroup of $\mathbb{Q}$ of finite index is $\mathbb{Q}$ itself we have $\phi(\mathbb{Q}) \subset K_{0}$. For $a \in A, a \neq 1$, we have $\phi\left(a^{n}\right) \in K_{0}$ for some $n$. Since $K_{0}$ is abelian, we have

$$
e=\phi\left(a^{n}\right) \cdot \phi(x) \cdot \phi\left(a^{-n}\right) \cdot \phi(-x)=\phi\left(\left(a^{n}-1\right) \cdot x\right)
$$

for every $x \in \mathbb{Q}$. Since every element of $\mathbb{Q}$ can be written in the form $\left(a^{n}-1\right) \cdot x$, we have

$$
\mathbb{Q} \subset \operatorname{ker} \phi \text {. }
$$

Conversely, $C / \mathbb{Q} \simeq A$ has an injective character.

\section{REFERENCES}

[1] H. Abels. Distal affine transformation groups. J. reine angew. Math. 299/300 (1978), 294-300.

[2] H. Abels. Distal automorphism groups of Lie groups. J. reine angew. Math. 329 (1981), 82-87.

[3] L. Auslander. An exposition of the structure of solvmanifolds. Bull Amer. Math. Soc. 79 (1973), 227-285.

[4] L. Auslander, L. Green \& F. Hahn. Flows on homogeneous spaces. Annals of Math. Studies 53. Princeton University Press: New Jersey, 1963.

[5] G. E. Bredon. Introduction to compact transformation groups. Academic Press: New York, 1972.

[6] R. Ellis. Distal transformation groups. Pacific I. of Math. 8 (1958), 401-405.

[7] H. Furstenberg. The structure of distal flows. Amer. J. Math. 85 (1963), 477-513.

[8] G. Higman. A finitely generated infinite simple group. Proc. London Math. Soc. 26 (1951), 61-64.

[9] G. Hochschild. The structure of Lie groups. Holden-Day, Inc: San Francisco, 1965.

[10] A. W. Knapp. Distal functions on groups. Trans. Amer. Math. Soc. 128 (1967), 1-40.

[11] A. I. Malcev. On faithful representations of infinite groups of matrices. Mat. Sb. 8 (1940), 405-422; (AMS Transl. (2) 45 (1965), 1-18.

[12] C. Moore \& R. Zimmer. Groups admitting ergodic actions with generalized discrete spectrum. Inv. math. 51 (1979), 171-188.

[13] J. Rosenblatt. A distal property of groups and the growth of connected locally compact groups. Mathematika 26 (1979), 94-98.

[14] R. Zimmer. Ergodic actions with generalized discrete spectrum. Illinois J. Math. 20 (1976), 555-588.

[15] R. Zimmer. Continuous ergodic extensions and fibre bundles. Can. J. Math. 30 (1978), 373-391. 